\title{
ON A CONVERSE OF THE HÖLDER INEQUALITY
}

\author{
ERNEST B. LEACH
}

Let $X$ be a measure space with measure $\mu$. We consider the associated dual metric spaces $L^{p}$ and $L^{q}$, where $1<p<\infty$, and $1 / p+1 / q$ $=1$. By the Hölder inequality, the product of an $L^{p}$ function with an $L^{q}$ function is integrable. The following converse is not always true: if $f$ is a measurable function whose product with every $L^{q}$ function is integrable, then $f$ belongs to $L^{p}$. The purpose of this note is to show that this converse is true for a given space $X$ if and only if every measurable subset $E$ of $X$, of measure $+\infty$, contains a measurable subset $F$, such that $0<\mu(F)<+\infty$.

It is useful to know that this condition implies the apparently stronger one: if $\mu(E)=+\infty$, then $E$ contains a measurable subset $F$ such that $\mu(F)=+\infty$, and $F$ is a countable union of sets of finite measure. To prove this, let $M=\sup \{\mu(F) \mid F \subset E \& \mu(F)<\infty\}$ (from here on we assume tacitly that all sets and functions referred to are measurable). Let $\left\{F_{n}\right\}$ be a sequence of subsets of $E$, such that $\mu\left(F_{n}\right)<\infty$ and $\lim _{n} \mu\left(F_{n}\right)=M$; and let $F=\bigcup_{n} F_{n}$. Then $\mu(F) \geqq M$, because $\mu(F) \geqq \mu\left(F_{n}\right)$ for all $n$. The stronger condition will be implied if we show that $\mu(F)=+\infty$. If not, then $\mu(E-F)=+\infty$, since $\mu(E)=+\infty$. By the assumed weaker condition on $X, E-F$ contains a subset $F^{\prime}$ of finite but positive measure. Then, if $\mu(F)<+\infty$, we have $M<\mu\left(F \cup F^{\prime}\right)<+\infty$, contrary to the choice of $M$.

To prove necessity of the condition, we note that if $X$ contains a subset $E$, of measure $+\infty$, whose subsets have measure 0 or $+\infty$, then the characteristic function of $E$ is evidently not $L^{p}$. But if $g$ is any $L^{q}$ function, the set $\{x \mid g(x) \neq 0\}$ is a countable union of sets of finite measure, and so intersects $E$ in a set of measure 0 . So the product of $g$ with the characteristic function of $E$ is 0 almost everywhere and thus is integrable, giving a denial of the converse of the Hölder inequality.

Before proving sufficiency, we shall show that if $f$ is any measurable function whose product with every $L^{a}$ function is integrable, and if $\{x \mid f(x)\} \neq 0$ is a countable union of sets of finite measure, then $f \in L^{p}$, independent of any condition on $X$. In this case there is a sequence, $\left\{f_{n}\right\}$, of functions each of which is bounded and vanishes except on a set of finite measure, and such that the sequence $\left\{\left|f_{n}\right|^{p}\right\}$ converges monotonely to $|f|^{p}$. Each of the functions $f_{n}$ determines a

Received by the editors May 9, 1955 and, in revised form, June 27, 1955. 
bounded linear functional on the space $L^{q}$, whose value for any $g$ in $L^{q}$ is given by $\left(f_{n}, g\right)=\int f_{n} g d \mu$, and whose norm is the $L^{p}$ norm of the function $f_{n}$, namely $\left(\int\left|f_{n}\right|^{p} d \mu\right)^{1 / p}$. Also for any $g$ fixed in $L^{q}$, the sequence of values of the functionals is bounded, since $\left|\left(f_{n}, g\right)\right|$ $\leqq \int|f g| d \mu<\infty$, because $\left|f_{n}\right| \leqq|f|$ and the product $f g$ is integrable by hypothesis. Therefore the Banach-Steinhaus theorem applies, and the sequence of norms of the functionals is bounded. Then $\int\left|f_{n}\right|{ }^{p} d \mu \leqq M$, and since $\left|f_{n}\right|^{p}$ converges monotonely to $|f|^{p}, \int|f|^{p} d \mu<\infty$. This proves the assertion. ${ }^{1}$

We can now prove sufficiency of the condition on $X$. Assuming this condition, let $f$ be any function whose product with every $L^{q}$ function is integrable. According to the preceding paragraph, we need only show that $\{x \mid f(x) \neq 0\}$ is a countable union of sets of finite measure. Let $E_{n}=\{x|| f(x) \mid \geqq 1 / n\}, n=1,2, \cdots$. We shall show that $\mu\left(E_{n}\right)<\infty$ for all $n$, which will prove the assertion. If not, then $\mu\left(E_{m}\right)=+\infty$ for some integer $m$, and by the "stronger" version of the condition on $X, E_{m}$ contains a subset $F$, such that $\mu(F)=+\infty$, and $F$ is a countable union of sets of finite measure. The product of $f$ and the characteristic function of $F$ satisfies the condition of the preceding paragraph and so belongs to $L^{p}$. But this is a contradiction because $|f| \geqq 1 / m$ on $F$, and $\mu(F)=+\infty$. This proves our theorem.

In closing we give a simple example of a measure space that satisfies our condition but which is not a countable union of sets of finite measure. If $X$ is any noncountable set, we assign to every finite subset of $X$ a measure equal to the number of points in that set; to all other sets we assign the measure $+\infty$.

Case Institute of Technology

1 This proof was given by Professor Walter Rudin in a course at Massachusetts Institute of Technology in 1951. The author is also indebted to the referee for observing that a proof for the case when $X$ is of finite measure may be found on p. 336 of Dunford, Uniformity in linear spaces, Trans. Amer. Math. Soc. vol. 44 (1938) pp. 305-356. 